\title{
Evaluation of thermal properties of thin films by IR radiometry using a comprehensive set of $\mathrm{Zr}-\mathrm{O}-\mathrm{N}$ thin films
}

\author{
C.I. da Silva-Oliveira ${ }^{1}$, D. Martinez-Martinez ${ }^{1}$, F. M. Couto ${ }^{2}$, L. Cunha ${ }^{1 *}$, F. Macedo ${ }^{1}$ \\ ${ }^{1}$ Center of Physics of the University of Minho and Porto, Braga, Portugal \\ ${ }^{2}$ Federal University of Espírito Santo, Chemistry Physics Dept., Campus Alegre, Brasil
}

*Corresponding author e-mail: lcunha@ fisica.uminho.pt

\begin{abstract}
Modulated Infrared Radiometry is a photothermal technique which allows thermal characterization of coatings. Thermal properties are determined by applying the "Extremum Method". Zr-O-N films were deposited by sputtering to evaluate their thermal properties and the sensitivity of the method and its suitability for different film+substrate systems. Three factors were varied: i) composition/bonding: metallic $\mathrm{Zr}$, crystalline metallic-type $\mathrm{Zr}-\mathrm{O}-\mathrm{N}$ and disordered ceramic $\mathrm{Zr}-\mathrm{O}$ $\mathrm{N}$. The films were deposited in the metallic, reactive and poisoned regimes of the hysteresis curve; ii) each film was deposited simultaneously on three different substrates: high-speed steel, glass and silicon; iii) in each deposition batch, films with four different thicknesses were grown. Each film was deposited in the same batch with different thicknesses on top of different substrates. All of the parameters for which the model is sensitive to are explored in this matrix of 36 different samples. The thermal parameters of the films were calculated and the trends and values were examined. The trends were explained in terms of the microstructural/chemical characteristics of the films, and the influence of each substrate, depending on the film thickness. The obtained values agree with those found in literature, reflecting the nature of the films.
\end{abstract}

Keywords: Zirconium oxynitride; Modulated Infrared Radiometry; Thermal conductivity; Volumetric heat capacity; Effusivity; Diffusivity

\section{Introduction}

Modulated infrared radiometry is a photothermal technique based on the creation of small temperature oscillations, usually called "thermal waves", by means of modulated heating of a 
material, and on the detection of the thermal response using an IR detector connected to a lock-in amplifier [1]. Once the penetration depth of the incident radiation can be controlled just by controlling the modulation frequency of the incident radiation, the technique is widely used for the thermal characterization of thin films and coatings [2]. This technique is also appropriate for remote detection in deposition chambers and industrial applications [3,4]. The amplitude and phase

6 lag of the recorded thermal wave contains all the information needed to determine the relevant thermal properties of a two layer system. Using an inverse solution of the two-layer thermal wave problem, direct relations can be established between the relative extrema of the inverse calibrated

9 thermal wave phase lag signals, measured as a function of the heating modulation frequency, and 10 fundamental thermal parameters of thin films and coatings, namely the ratio of the thermal 11 effusivities coating-to-substrate, the coating's thermal diffusion time, and the coating thickness 12 [4].

The goal of this work is twofold. First, to obtain the thermal characteristics of the three different types of $\mathrm{Zr}-\mathrm{O}-\mathrm{N}$ films, deposited in the metallic, reactive and poisoned regimes of the hysteresis curve. Second, to test the sensitivity of the technique to all the parameters included in the two-layer model, by taking advantage of the systematic approach employed during the film

17 deposition which led to three different $\mathrm{Zr}-\mathrm{O}-\mathrm{N}$ films deposited during four deposition times (from 181 to 4 hours) on top of three different substrates (high speed steel, silicon and glass).

\section{Experimental details}

\section{$21 \quad$ 2.1. Thin film deposition and characterization}

$\mathrm{Zr}-\mathrm{O}-\mathrm{N}$ thin films were deposited onto $(111)$ silicon pieces $(1.5 \mathrm{~cm} \times 1.5 \mathrm{~cm})$, glass $(2 \mathrm{~cm}$ $\times 2 \mathrm{~cm})$ and mirror-polished high-speed steel (HSS) cylindrical substrates $(\varnothing=3 \mathrm{~cm} \times 0.5 \mathrm{~cm})$ by reactive direct current magnetron sputtering in a laboratorial size deposition equipment. The substrates were first cleaned with ethanol and etched in a Zepto Plasma System (Diner) equipped with a $40 \mathrm{kHz} / 100 \mathrm{~W}$ generator. During the etching process, the power used was $100 \mathrm{~W}$ and the Ar pressure was approximately $80 \mathrm{~Pa}$. For the depositions, the substrates were clamped in a rotating holder $(5 \mathrm{rpm})$ placed at $75 \mathrm{~mm}$ from the magnetron head. The base pressure was always below $2.6 \times 10^{-3} \mathrm{~Pa}$. The depositions were performed by sputtering of a $\mathrm{Zr}$ target $(99.6 \%$ at., $10 \times 20$ $\mathrm{cm}^{2}$ ) using $\mathrm{Ar}$ as working gas and $\mathrm{N}_{2}$ and $\mathrm{O}_{2}$ as reactive gases. During the deposition, the discharge parameters (target potential, applied current and working pressure) were monitored using a Data 
Acquisition/Switch Unit Agilent 34970A, with a multifunction module. This unit uses a RS-232 interface and the data is acquired with a Benchlink Data Logger III software.

In previous papers the influence of different deposition parameters (e.g. flow of the reactive mixture, bias, doping with Ti...) in the characteristics and optical properties of $\mathrm{Zr}-\mathrm{O}-\mathrm{N}$ films has been investigated[5,6]. Three different sets of synthesis conditions (Metallic, Reactive and

6 Poisoned, henceforth referred as M, R and P films, respectively) were distinguished, which lead to 3 clear types of films: metallic $\mathrm{Zr}$ (metallic grey color, films M), crystalline $\mathrm{Zr}$-O-N (golden, films R) and disordered $\mathrm{Zr}-\mathrm{O}-\mathrm{N}$ (transparent to visible radiation, films $\mathrm{P}$ ). These conditions were chosen to deposit three different sets of films using different $\mathrm{N}_{2}$ and $\mathrm{O}_{2}$ flows (see Table 1), while maintaining the remaining conditions constant (Ar flow $18 \mathrm{sccm}, \mathrm{Zr}$ target current $2 \mathrm{~A}$ ).

Additionally, for each of the three sets of films four different deposition times were used. In this manner 36 different samples were deposited (3 different film types, 4 different thicknesses and 3 different substrates). To optimize the depositions and keep the conditions of the films of the same type (M, R or P) exactly the same, the samples with different deposition times were deposited in the same batch. To do that, the substrates were clamped on four faces of the holder, and three of them were covered with a metallic shield at the beginning of the deposition (see Figure 1a). The deposition of each type of film was carried on during four hours, and one of the shields was removed after each hour in order to expose a new set of substrates ( $\mathrm{Si}$, glass and steel) to the plasma. At the end, four different thicknesses were obtained for each deposition batch, as summarized in the scheme of Figure 1b. The thickest films were growing for 4 hours, while the thinnest ones grew only for the last hour of the deposition process, which constitutes the top part of the thicker films (Figure 1b).

The chemical composition of the films was determined by Rutherford backscattering spectrometry (RBS) on the samples deposited during $2 \mathrm{~h}$, using CTN/IST Van der Graaff accelerator in a small chamber were tree detectors were installed: standard at $140^{\circ}$, and two pindiode detectors located symmetrically to each other both at $165^{\circ}$. The spectra were collected for 2 . $\mathrm{MeV}^{4} \mathrm{He}^{+}$beam and the angle of incidence was $0^{\circ}$ (normal incidence). The compositional profile of the samples was determined using the software IBA Data furnace NDF v9.6i [7], double scattering and Pileup were calculated using the algorithms given elsewhere[8,9]. The density and thickness of the films have been also calculated. The chemical composition was also investigated 31 through Energy-dispersive X-ray spectroscopy (EDX) for the samples deposited during $1 \mathrm{~h}$ and $4 \mathrm{~h}$ in an EDAX -Pegasus X4M (EDS/EBSD) equipment operating at $10 \mathrm{kV}$. 
The morphology of the films was characterized by scanning electron microscopy (SEM)

2 in a FEI - Nova 200 NanoSEM (FEG/ESEM) equipment operating at $10 \mathrm{kV}$ in the samples 3 deposited during $1 \mathrm{~h}$ and $4 \mathrm{~h}$.

4 The crystallographic structure was investigated by X-ray diffraction in grazing incidence

$5 \quad 4^{\circ}$ on a Brucker D8 Advanced system apparatus using $\mathrm{Cu} \mathrm{k}_{\alpha}$ radiation $(\lambda=0.154 \mathrm{~nm})$ in the samples

6 deposited during $3 \mathrm{~h}$.

\subsection{Modulated IR Radiometry and determination of thermal parameters}

9 Thermal properties determination was performed using data obtained by Modulated IR 10 Radiometry (MIRR), a non-contact and non-destructive photothermal technique based on the 11 response from the materials to an intensity-modulated laser beam irradiation[1,10]. The created

12 "thermal waves" are then detected using an IR HgCdTe detector, connected to a two-phase Lock13 in amplifier (SR830), used to filter and amplify the small periodical variations of the detected IR 14 emission caused by the time and space small temperature oscillations occurring in the samples.

15 These small non-continuous oscillations allow us to separate the useful information from the high 16 radiation and temperature background around $300 \mathrm{~K}$. Due to the frequency dependence "thermal 17 thickness" control, this technique is particularly suitable for studying thin films and coatings [1,2], 18 once depth resolved measurements of the thermal properties of thin films or coatings deposited on 19 thermally thick substrates can be done. Detailed information on the experimental setup can be 20 found elsewhere [11].

21 In order to interpret the signals measured for two-layer systems, like the ones studied here, 22 the in-phase and out-of-phase components of the modulated IR signals, giving information on the 23 thermal waves' amplitude and phase lag relative to the modulated excitation were measured by a

24 Lock-in amplifier. These measurements were normalised with the help of thermal wave signals 25 measured for a homogeneous opaque reference sample (sigradur®) under the same conditions.

26 This normalization eliminates all the frequency characteristics of the various components of the 27 measurement system.

28 The thermal diffusion time of the thin films, and the effusivity ratio of the coating/substrate 29 system can be obtained by applying the so-called "extremum method" [4]. These are fundamental 30 parameters to understand the role of heat diffusion and propagation on thin films and coatings.

31 According to this method, analytical solutions can be found for the relative extrema (i.e. minimum 
1 or maximum) of the normalized phase $\left(\Phi_{\mathrm{n}}\right)$ vs $(f / \mathrm{Hz})^{1 / 2}$. This results in unique solutions for the

2 coating-to-substrate thermal reflection coefficient $\left(R_{c s}\right)$ and the coating's thermal diffusion time

$3\left(\tau_{\mathrm{c}}\right)$, as follows:

$4 \quad R_{C S}= \pm \sqrt{\frac{1-\tan \theta}{1+\tan \theta}} \exp \theta$

$5 \quad \tau_{c}=\frac{\theta^{2}}{4 \pi f_{\text {extr }}}$

$6 \quad$ where $\theta=\frac{1}{2} \operatorname{arcos}\left(\tan \Phi_{n, e x t r}\right)^{2}$

$7 \quad$ From equations (1-3), the thermal reflection coefficient $\left(R_{c s}\right)$ and the thermal diffusion time

$8\left(\tau_{c}\right)$ can be calculated directly from the measured quantities $\Phi_{n, \text { extr }}$ and $f_{\text {extr }}$, which represent the

9 minimum value of the normalized phase and the frequency at which the minimum occurs,

10 respectively. Once the thickness $\left(d_{c}\right)$ of the films were independently measured by SEM, the

11 thermal diffusivity of the films can also be calculated[3]:

$12 \quad \alpha_{\mathrm{c}}=d_{\mathrm{c}}{ }^{2} / \tau_{\mathrm{c}}$

13 Finally, provided that the thermal effusivity of the substrate $\left(e_{s}\right)$ is known, the thermal

14 effusivity of the film $\left(e_{c}\right)$ can be calculated from the film to substrate effusivity ratio $\left(R_{c s}\right)$ :

$15 R_{C S}=\frac{e_{c}-e_{s}}{e_{c}+e_{s}}$

16 The thermal diffusivity and effusivity of the coating are functions of its thermal 17 conductivity $\left(k_{c}\right)$, density $\left(\rho_{c}\right)$ and specific heat capacity $\left(C_{c}\right)$. The product of these latter two 18 magnitudes $(\rho C)_{c}$ is the volumetric heat capacity of the coating, which can be calculated for the 19 coating together with its thermal conductivity using these expressions:

$20 \quad \alpha_{c}=\frac{k_{c}}{(\rho C)_{c}}$

$21 e_{c}=\sqrt{k_{c}(\rho C)_{c}}$

22 It is worth mentioning that the coordinates of the extremum $\left(f_{\text {extr }}, \Phi_{n, \text { extr }}\right)$ are not directly

23 obtained from the experimental data point with the lowest value of $\Phi_{n, \text { extr }}$ Instead, we performed

24 a fitting around the location of extremum using the general function $\Phi_{n}(f)$, which can be expressed 25 as:

$26 \tan \Phi_{n}(f)=\frac{2 R_{c s} \exp \left(-2 \sqrt{\pi \cdot f \cdot \tau_{c}}\right) \sin \left(2 \sqrt{\pi \cdot f \cdot \tau_{c}}\right)}{1-\left[R_{c s} \exp \left(-2 \sqrt{\pi \cdot f \cdot \tau_{c}}\right)\right]^{2}}$ 
This approach has two advantages. First, the error is reduced, since the extremum is located

2 by considering the shape of the curve and thus with several experimental data instead only one.

3 Second, the determination of $f_{\text {extr }}$ is no longer restricted to the discrete values of frequency selected

4 for the experimental measurements, but it is obtained from the location of the extremum of the

5 fitting curve, which is not conditioned by the experimental conditions.

3. Results and discussion

\subsection{Characteristics of the films}

The characteristics of the deposition processes and the films are included in Table 1. The

10 values of pressure and voltage show a certain degree of drift during each deposition, which is

11 reasonable considering the duration of the deposition process. However, EDX measurements

12 carried out in the thinnest and thickest films are very similar, indicating that the chemical

13 composition of the films remains invariant through their thickness. Nevertheless, it can be noticed

14 a large overestimation of the $\mathrm{N}$ content by EDX for the film $\mathrm{M}$, which was produced without any

15 introduction of $\mathrm{O}_{2}$ nor $\mathrm{N}_{2}$. This is pointed out by comparison with the RBS measurements carried

16 out on this sample ( $7 \%$ of $\mathrm{O}$, and absence of nitrogen).

17 Figure 2 shows $\mathrm{X}$-section SEM images of the three types of films (M, R, and P) deposited

18 at 1 and 4 hours. Both $\mathrm{M}$ and $\mathrm{R}$ samples have a columnar morphology characteristic from PVD

19 deposited films, as observed in the specimens grown for $1 \mathrm{~h}$. The width of the columns decreases

20 with the increase of the flow of the reactive gas mixture (Figure 2a and b). The samples deposited

21 with the highest flow present a denser microstructure (Figure 2c). This evolution is very similar to

22 what has been reported by other authors[12,13]. For the samples deposited for $4 \mathrm{~h}$ the evolution of

23 the microstructure is similar, but the samples identified previously as columnar tend to evolve to

24 a cone-like shape structure it the top part of the film. Huang et al also observed the formation of

25 this cone-shape microstructure for samples deposited during $2 \mathrm{~h}$ [14]. The sample in the poisoned

26 region still presents a dense microstructure. It is worth mentioning that many of the features of the

27 thicker films (e.g. columns) grow through the whole thickness of the film, despite the 3

28 interruptions suffered during the deposition due to the removal of the shields that protect the 29 substrates. 
The characteristics of these films are in agreement with the three regions observed in

2 previous works [5,6]. Thus, film $M$ has metallic grey colour and it shows a clear metallic composition together with a XRD pattern composed by peaks of metallic $\alpha-\operatorname{Zr}$ (ICCD 03-0653366) (see Figure 3). Film $\mathrm{R}$ is golden, and it has a $\mathrm{Zr} / \mathrm{N}$ ratio close to 1 , with some addition of $\mathrm{O}$ (ca. 15 at. \%). It is composed by sharp peaks of c- $\mathrm{ZrN}$ and c- $\mathrm{Zr}_{2} \mathrm{ON}_{2}$ (ICCD 03-065-7723 and 01089-8345 respectively) (see Figure 3). Finally, film P, although chemically similar to the second, shows a $\mathrm{Zr} / \mathrm{N}$ ratio more deviated from stoichiometry (1.3), and a higher O concentration (19 at. $\%)$. As a result, the structure is more amorphous, presenting broader peaks due to the distortion of the Zr-O-N lattice (see Figure 3).

\subsection{Thermal properties}

The experimental results presented herein refer only to the phase lag $\left(\Phi_{n}\right)$ vs. frequency $(f)$

13 data from which the thermal parameters can be immediately determined, as explained in Section

14 2.2. Figure 4 shows the experimental data obtained for the inverse normalized phase lag vs 15 modulation frequency for three different types of thin films deposited onto three different 16 substrates with four different deposition times. The acquired experimental values and simulations 17 according to the opaque-opaque two-layer model are represented by discrete symbols and solid 18 lines, respectively.

The results are organized by showing the three different substrates (steel, silicon and glass) in rows and the three types of films (M, R and P) in columns. Films $\mathrm{P}$ become transparent for visible radiation as a consequence of the high $\mathrm{O}$ content. As a consequence, the results present deviations from the zero line of the inverse normalized phase lag (cf. Figs 4c, f and i). These results are not predictable from the theoretical model we are currently using, since it does not consider a high degree of semi-transparency of the film. A generalization of the theoretical model, including the semi-transparency approach, is underway.

In each of the 9 substrate-film intersections there are 4 sets of data that represent films with deposition times between 1 and 4 hours. In that regard, according to Eqs.1-5, if we consider a pure variation of film thickness $\left(d_{c}\right)$ while keeping the film and substrate parameters invariant, we

29 should expect a shift of the position of the extremum $\left(f_{\min }\right)$ towards higher values (caused by the 30 reduction of $\tau_{c}$ ), but not a change in the value of $\Phi_{n, \min }$. This is quite the case in few cases in Figure 314 (see e.g. Fig. 4c), although this is not the general behavior. In contrast, it is much more common 
that the height of the extreme is also changed (see for instance Fig. 4a), which indicates a variation

2 of the thermal properties of the film for different thicknesses. In addition, in some of the samples, 3 it was not possible to find a clear minimum or maximum (films R1 and P1 on steel, P1 on silicon 4 and films $\mathrm{P}$ on glass). Further, uncommon narrow shapes of the plots have been identified in two cases (films M1 on silicon and R4 on glass). These behaviors can be related with issues during measurement or adhesion problems. Therefore, although the fitting results and thermal properties of all the films are summarized in Table 2, the data from these samples found clearly out of range has been excluded from the plots of the behavior of the thermal properties of the films depending on their thickness, which are depicted in Figure 5.

The main general observation is that there is much lower dispersion in the results of the

11 films $\mathrm{P}$ (right column in Fig. 5) than in those obtained for films $\mathrm{M}$ and R. It is worth mentioning 12 that such effect cannot be attributed to the exclusion of the films P deposited on glass, since the 13 dispersion of data considering only films deposited on silicon and steel is still much higher for

14 films M and R. This result is likely connected to the different nature of the films; thus, films M 15 and $\mathrm{R}$ are crystalline and show a clear columnar growth, while films $\mathrm{P}$ are amorphous and much 16 denser. As a consequence, this latter film seems to grow similarly onto different substrates and to 17 show similar properties regardless of the thickness. In other words, little microstructural variation 18 is observed for this film (the film is 'always the same').

The values of effusivity ratio $\left(e_{d} / e_{s}\right)$ for films deposited on steel and silicon are lower than 1, and the opposite is observed in case of glass (cf. top row in Fig. 5). This is expected due to the different nature of the substrates in comparison with the films, and in agreement with the type of extremum (minimum or maximum) observed in Figure 4. The values are not constant, indicating that the thermal properties of the film will depend on the substrate and the thickness of the film.

24 The trends of thermal diffusivity, conductivity, and volumetric heat capacity of the coating $\left(\alpha_{c}, k_{c}\right.$, and $\left.(\rho C)_{c}\right)$ with film thickness are similar for the three films, although clearer for films $\mathrm{M}$ and $\mathrm{R}$ than for $\mathrm{P}$ ones, probably due to the reasons explained above. In general, $k_{c}$ and $\alpha_{c}$ show a growing trend with film thickness, while the opposite is seen for $(\rho C)_{c}$; in all the cases, a kind of 'steady state' is reached for the thickest films. The reason for this 'stabilization' is likely due to the microstructural evolution of the films, which is has more weight when changing from $1 \mathrm{~h}$ to $2 \mathrm{~h}$ of deposition time (thickness increases by ca. 100\%) than when changing from 2 to $3 \mathrm{~h}$ (increase 31 of ca. $50 \%$ ) or from 3 to $4 \mathrm{~h}$ (increase of ca. $33 \%$ ). In other words, the film moves towards a 'bulk' 
state, but the rate of variation of film properties reduces. The increase of thermal conductivity can be well explained in terms of microstructural variation (improved interconnection, filling of 3 spaces), while the reduction of volumetric heat capacity could be related to the reduction of density 4 for thicker films (larger voids and large column separation). Wei et al. [15], reported an exponential 5 increase of $\alpha$ for different substrates. This behavior was also observed in some cases, e.g. films M on steel (linear behavior on Fig. 5d, notice the logarithmic y axis). These authors address other two factors to explain this trend. First, the presence of interfacial thermal resistance caused by the mismatch in thermal conductivity between film and substrate, which alters the heat flow and the resultant $\alpha$ of the combined system. This effect would be reduced for higher film thickness, due to the annealing effects caused by longer duration of deposition. Second, the presence of grain 11 boundaries in microstructured thin films can cause scattering of the phonons, which carry the thermal energy. This effect will be reduced in in thicker films due to the coalescence of grains.

Beyond the general observed trends, a certain dispersion of data is observed when

14 comparing results of films on different substrates (i.e. different values may be obtained for a certain 15 film with a certain thickness in different substrates). However, it seems that such dispersion decreases with thickness of the films. This can be easily observed for films R (cf. e.g. Fig. 5e).

17 This can be also appreciated for the films M, although the films M2 and M3 on Si show values 18 that seem out of the trend, likely connected to the noisy results in Fig. 4d. The reason for the reduction of data dispersion with thickness likely reflects that thin films are more influenced by substrate (e.g. different types of film growth, wetting, etc.), but that influence gets reduced when 21 the thickness increases.

The value of $\alpha_{c}$ for the films M is ca. $10^{-6} \mathrm{~m}^{2} / \mathrm{s}$, which fits very well with values found for $23 \mathrm{Zr}$ thin [16] and also for bulk $\mathrm{Zr}\left(12.4 \times 10^{-6} \mathrm{~m}^{2} / \mathrm{s}\right)$, since for metallic thin films the thermal 24 diffusivity values are typically one order of magnitude below those corresponding to the bulk 25 material. The values of $\alpha_{c}$ in steel are in agreement with other results $\mathrm{Zr}-\mathrm{O}-\mathrm{N}$ films[11,17]. In fact, 26 it is observed that $\alpha_{c}$ varies from ca. $10^{-6}$ to $5 \times 10^{-7} \mathrm{~m}^{2} / \mathrm{s}$ when changing the nature of the film from 27 M to R to P (considering the 'steady state' values), in excellent agreement with the results 28 previously reported [17]. The values of $k_{c}$ indicate low thermal conductivity, probably related with 29 the columnar microstructure of the films. However, the values of the P film are lower than $\mathrm{M}$ and $30 \mathrm{R}$ (considering the steady state values), in agreement with the chemical nature of the films (metals 
vs. ceramic). The values obtained for $(\rho C)_{c}$ are also in the good range, which is supposed to be

2 between $10^{6}$ and $10^{7} \mathrm{~J} / \mathrm{m}^{3} \mathrm{~K}[1]$.

\section{Conclusions and outlook}

A systematic study of thermal properties of $\mathrm{Zr}-\mathrm{O}-\mathrm{N}$ thin films was carried out, using a modulated IR radiometry. Three sets of films were used: metallic type (M); oxynitride reactive type (R) and oxynitride poisoned type (P). The films of each one of these sets had the same composition (deposited in the same batch), but they were deposited on three different substrates and four different thicknesses in each type of substrate. This systematic study allowed us to test the accuracy of the Extremum method when varying different experimental parameters.

In general, the results have been quite satisfactory, and the different observations have been

12 successfully explained based on the phase and microstructural characteristics of the films. P shows

13 a much lower dispersion of data, as a consequence of its amorphous nature and dense growth, in

14 opposition to films $\mathrm{M}$ and $\mathrm{R}$. These two films show a variation of thermal properties with the

15 thickness of the film that could be addressed in terms of microstructural variation (e.g. better 16 columnar interconnection). Additionally, the dispersion of data among substrates was higher at 17 lower thickness, probably due to higher influence of substrate (e.g. growth type and wetting). 18 Finally, the values of the thermal parameters are in agreement with results from literature, and in 19 line with the chemical nature of the films.

\section{Acknowledgements}

22 This work was supported by the Portuguese Foundation for Science and Technology (FCT) under 23 the project number IF/00671/2013, M-ERA.NET2/0012/2016 and the framework of the Strategic 24 Funding UID/FIS/04650/2013.

\section{References}

27 [1] D.P. Almond, P. Patel, Photothermal Science and Techniques, Springer, 1996.

28 [2] F. Macedo, A. Gören, F. Vaz, J.L. Nzodoum Fotsing, J. Gibkes, B.K. Bein, Photothermal characterization of thin films and coatings, 82 (2008) 1461-1465. doi:10.1016/j.vacuum.2008.03.007. 
[3] M. Apreutesei, C. Lopes, J. Borges, F. Vaz, F. Macedo, Modulated IR radiometry for determining thermal properties and basic characteristics of titanium thin films, J. Vac. Sci. Technol. A Vacuum, Surfaces, Film. 32 (2014) 41511. doi:10.1116/1.4884351. J.L.N. Fotsing, J. Gibkes, J. Pelzl, B.K. Bein, Extremum method: Inverse solution of the two-layer thermal wave problem, J. Appl. Phys. 98 (2005) 63522. doi:10.1063/1.2058180. C.I. da Silva Oliveira, D. Martinez-Martinez, A. Al-Rjoub, L. Rebouta, R. Menezes, L. Cunha, Development of a statistical method to help evaluating the transparency/opacity of decorative thin films, Appl. Surf. Sci. 438 (2018) 51-58. doi:10.1016/j.apsusc.2017.10.017.

6] C.I. da S. Oliveira, D. Martinez-Martinez, L. Cunha, M.S.S. Rodrigues, J. Borges, C. Lopes, E. Alves, N.P.P. Barradas, M. Apreutesei, C.I. da Silva Oliveira, D. Martinez-Martinez, L. Cunha, M.S.S. Rodrigues, J. Borges, C. Lopes, E. Alves, N.P.P. Barradas, M. Apreutesei, $\mathrm{Zr}-\mathrm{O}-\mathrm{N}$ coatings for decorative purposes: Study of the system stability by exploration of the deposition parameter space, Surf. Coatings Technol. In Press (2018) 1-8. doi:10.1016/j.surfcoat.2017.11.056.

[7] N.P. Barradas, C. Jeynes, R.P. Webb, U. Kreissig, R. Grötzschel, Unambiguous automatic evaluation of multiple Ion Beam Analysis data with Simulated Annealing, Nucl. Instruments Methods Phys. Res. Sect. B Beam Interact. with Mater. Atoms. 149 (1999) 233-237. doi:10.1016/S0168-583X(98)00731-9.

8] N.P. Barradas, C. Pascual-Izarra, Double scattering in RBS analysis of PtSi thin films on Si, Nucl. Instruments Methods Phys. Res. Sect. B Beam Interact. with Mater. Atoms. 228 (2005) 378-382. doi:10.1016/j.nimb.2004.10.074.

[9] N.P. Barradas, M.A. Reis, Accurate calculation of pileup effets in PIXE spectra from first principles, X-Ray Spectrom. 35 (2006) 232-237. doi:10.1002/xrs.

[10] C.A. Bennett, R.R. Patty, Thermal wave interferometry: a potential application of the photoacoustic effect, Appl. Opt. 21 (1982) 49-54. doi:10.1364/AO.21.000049.

[11] J. Gibkes, F. Vaz, a C. Fernandes, P. Carvalho, F. Macedo, R.T. Faria, P. Kijamnajsuk, J. Pelzl, B.K. Bein, Analysis of multifunctional oxycarbide and oxynitride thin films by modulated IR radiometry, J. Phys. D. Appl. Phys. 43 (2010) 395301. doi:10.1088/00223727/43/39/395301.

[12] S.C. Ferreira, E. Ariza, L.A.A. Rocha, J.R.R. Gomes, P. Carvalho, F. Vaz, a. C.C. Fernandes, L. Rebouta, L. Cunha, E. Alves, P. Goudeau, J.P.P. Rivière, Tribocorrosion behaviour of $\mathrm{ZrOxNy}$ thin films for decorative applications, Surf. Coatings Technol. 200 
(2006) 6634-6639. doi:10.1016/j.surfcoat.2005.11.083.

[13] J.-H. Huang, T.-H. Wu, G.-P. Yu, Heat treatment induced phase separation and phase transformation of $\mathrm{ZrNxOy}$ thin films deposited by ion plating, Surf. Coatings Technol. 203 (2009) 3491-3500. doi:10.1016/j.surfcoat.2009.05.016.

[14] J.-H. Huang, Y.-Y. Hu, G.-P. Yu, Structure evolution and mechanical properties of ZrNxOy thin film deposited on Si by magnetron sputtering, Surf. Coatings Technol. 205 (2011) 5093-5102. doi:10.1016/j.surfcoat.2011.05.015.

[15] P.C. Wei, H.C. Shih, S. Chattopadhyay, C.M. Hsu, F.S. Lin, K.H. Chen, A. Ganguly, L.C. Chen, Thermal diffusivity study in supported epitaxial $\mathrm{InN}$ thin films by the traveling-wave technique, J. Appl. Phys. 104 (2008). doi:10.1063/1.2986314.

[16] A. Mandelis, ed., Progress in Photothermal \& Photoacoustic Science \& Technology, Vol 1 Principles and perspectives of photothermal and photoacoustic phenomena, Elsevier, New York, 1992.

[17] F. Macedo, P. Carvalho, L. Cunha, F. Vaz, J. Gibkes, B.K. Bein, J. Pelzl, F. Macedo, P. Carvalho, B.K. Bein, J. Pelzl, The Role of Modulated IR Radiometry Measurements in the Characterization of Zr-O-N Thin Films, Plasma Process. Polym. 6 (2009) S760-S766. doi:10.1002/ppap.200931802. 


\section{Figures captions}

Figure 1. a) Representation of the deposition chamber setup. 1 - Magnetron head, 2 - Target, 3Substrate holder, 4 - Cover; 5- Substrates (glass, steel and silicon pieces). b) Scheme of the growth of films with different thickness by exposing the substrates to different deposition times (1, 2, 3 and 4 hours). The indexes I, II, III and IV represent growth occurring during the first, second, third, and fourth hours of deposition, respectively.

Figure 2 .X-section SEM images of different Zr-O-N films. a) M1. b) R1. c) P1. d) M4. e) R4. f) P4. All the scale bars represent $500 \mathrm{~nm}$.

Figure 3. X-ray diffraction of representative films of each type and respective phase identification. For the film M, an inset is included to zoom in the peaks with lower intensity.

Figure 4. Inverse calibrated IR phase lag signals measured for three types of films (M, R and P), on three different substrates (steel, silicon and glass) with four different deposition times. The points and solid lines correspond to experimental measurements and the opaque two-layer approximations (Eq. 8), respectively.

Figure 5. Thermal parameters of the different samples obtained from the fittings of the experimental results with the two layers model represented in Figure 4 using Equations 1-8. The black dashed lines represent guides for the eye. 